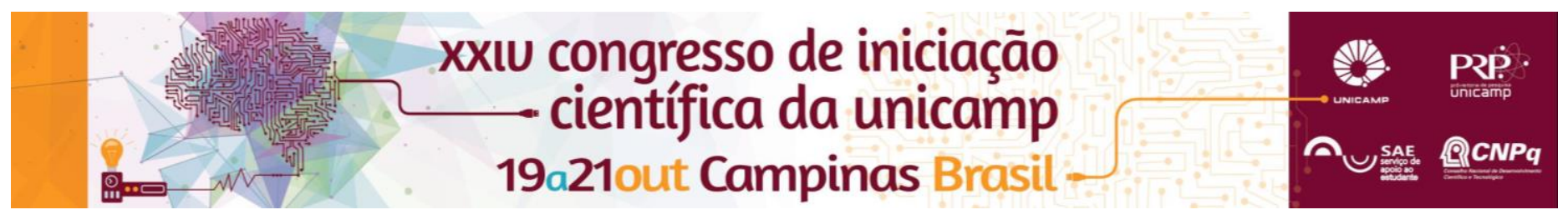

\title{
Effectiveness of four methods of teeth sterilization and their influence on the proteolytic activity of bovine dentin
}

\author{
Jina Sung ${ }^{\star}$, Jossaria P. de Sousa, Luiz Filipe Barbosa-Martins, Regina Maria Puppin-Rontani
}

\begin{abstract}
This study evaluated the effectiveness of methods commonly used for sterilization of teeth and their influence on proteolytic activity of bovine dentin. Bovine dentin blocks were contamined with $\mathrm{E}$. faecalis suspension for $24 \mathrm{~h}$, and then randomized into four groups ( $n=5)$ : $G 1$ - gamma radiaton (GR); $G 2$ - ultraviolet light (UV); $G 3$ - ethylene oxide (EO), and G4 - autoclaving (AC). Effectiveness of methods was verified based on the growth of microorganisms on blood agar culture medium, and the proteolytic activity of dentin assessed by in situ Zymography. No microbial growth was observed for GR, OE and AC groups, while UV was not capable to reduce CFUs of E. faecalis. GR, EO and AC increased the proteolytic activity of MMPs, in contrast to UV method. It could be concluded that the methods considered effective for teeth sterilization affected the proteolytic activity of bovine dentin.
\end{abstract}

Key words: Sterilization; dentin; matrix metalloproteinase 2

\section{Introduction}

In vitro and in situ researches that use human or bovine extracted teeth normally require that teeth being sterilized, in order to avoid cross infection (Kumar et al., 2005). Autoclaving, gamma radiation, and ethylene oxide have been described in the literature as methods capable of decontaminate dental substrates (Sandhu et al., 2012). However, few evidence has been found about the effect of these methods on the organic content of dentin. Then, the current study aimed to evaluate the effectiveness of methods commonly used for sterilization of teeth, and to assess their effects on proteolytic activity of bovine dentin.

\section{Results and Discussion}

\section{Flow-chart of the study design}

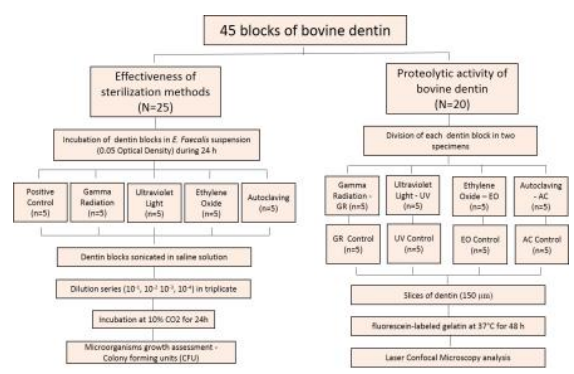

\section{Effectiveness of methods used for teeth sterilization}

No microbial growth was observed for gamma radiation or ethylene oxide methods, similarly to autoclaving. However, irradiation with ultraviolet light was not capable to eliminate or reduce CFUs of $E$. faecalis (Table 1).

Table 1. Mean and standard deviation (SD) of CFU/ml count in each sterilization group.

\begin{tabular}{lcc}
\hline & \multicolumn{2}{c}{ CFU/ml } \\
\cline { 2 - 3 } Groups & Mean & Standard deviation \\
\hline Positive Control & $4.0 \times 10^{5}$ & $0.4 \times 10^{5}$ \\
Gamma Radiation & 0.0 & 0.0 \\
Ultraviolet Light & $3.6 \times 10^{5}$ & $0.5 \times 10^{5}$ \\
Ethylene Oxide & 0.0 & 0.0 \\
Autoclaving & 0.0 & 0.0 \\
\hline
\end{tabular}

\section{Proteolytic activity of bovine dentin}

Gamma radiation, ethylene oxide and autoclaving methods increased the proteolytic activity of bovine dentin when compared to their controls. In contrast, irradiation with ultraviolet light did not cause any change on MMPs activity, as showed in Figure 1.

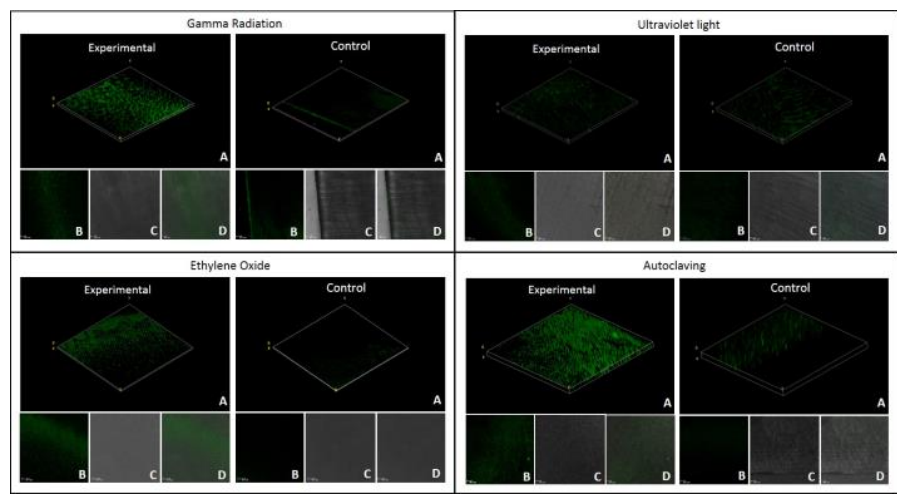

Figure 1. Representative images of proteolytic activity of dentin after had submitted to different methods of sterilization. A- 3D reconstruction. B- Acquired images in green channel presenting the fluorescence of dentin. C- Differential interference contrast (DIC) showing the optical density of dentin. D- Merged images of B and C.

\section{Conclusions}

It could be concluded that the same methods considered effective for teeth sterilization (gamma radiation, ethylene oxide and autoclaving) affected negatively the proteolytic activity of bovine dentin.

\section{Acknowledgement}

The authors thank CNPq and FAPESP (number protocol: 2014/22899-8) for financial support of this research.

Kumar, M.; Sequeira, P.S.; Peter, S.; Bhat, G.K. Sterilisation of extracted human teeth for educational use. Indian J. Med. Microbiol. 2005.

Sandhu, S.V.; Tiwari, R.; Bhullar, R.K.; et al. Sterilization of extracted human teeth: A comparative analysis. J Oral Biol Craniofac Res. 2012; 2(3):170-175. 